\title{
Expression and Potential Role of microRNA-29b in Mouse Early Embryo Development
}

\author{
Junqiang Zhang ${ }^{a, b}$ Ying Wang ${ }^{a}$ Xiaoguang Liu ${ }^{a}$ Shenglin Jiang ${ }^{a}$ Chun Zhao \\ Rong Shen ${ }^{a}$ Xirong Guo Xiufeng Ling ${ }^{a}$ Chang Liub \\ aDepartment of Reproduction, Nanjing Maternity and Child Health Care Hospital, Affiliated to Nanjing \\ Medical University, Nanjing Medical University, Nanjing, bJiangsu Key Laboratory for Molecular and \\ Medical Biotechnology, College of Life Sciences, Nanjing Normal University, Nanjing, China
}

\section{Key Words}

MiR29b • Dnmt3a/3b • DNA Methylation • Early Embryonic Development

\begin{abstract}
Background/Aims: MicroRNA-29b (miR29b) has been previously identified in early mouse embryos through miRNA microarray analysis. Recent research has indicated that miR29b participates in DNA methylation by regulating DNA methyltransferase 3a/3b (Dnmt3a/3b) expression. However, the expression pattern and biological function of miR29b in mouse preimplantation embryonic development remain unknown. Methods: In this study, we examined the expression patterns of miR29b and Dnmt3a/3b in mouse early embryos at different developmental stages. Subsequently, expression and localization of DNMT3A/3B protein was analyzed in mouse early embryos by immunofluorescence staining. The biological function of miR29b in mouse early embryos was analyzed by microinjection of commercially available miRNA-specific inhibitors and mimics. Results: Our data showed that Dnmt3a/3b mRNA expression is negatively regulated by miR29b in mouse early embryos. Immunofluorescence analysis revealed that DNMT3A/3B protein expression is predominantly localized within the nucleoplasm of embryos. Alterations to the activity of miR29b could change the DNA methylation levels in mouse preimplantation embryos and lead to a developmental blockade, from the morula to the blastocyst stage. Conclusion: These results indicated a role for the miR29b-Dnmt3a/3b-DNA methylation axis in mouse early embryonic development, and we provide evidence that miR29b is indispensable for mouse early embryonic development. This study contributes to a preliminary understanding of the role of miR29b during mouse embryonic development.
\end{abstract}

J. Zhang and Y. Wang have contributed equally to this work.

Xiufeng Ling,

Chang Liu

\section{KARGER 125}

Department of Reproduction, Nanjing Maternity and Child Health Hospital, Nanjing Medical University, 123 Mochou Road, Nanjing 210004, (P.R. China) and Jiangsu Key Laboratory for Molecular and Medical Biotechnology, College of Life Sciences, Nanjing Normal University, 1 WenYuan Road, Nanjing, Jiangsu 210046, (P.R. China)

E-Mail lingxiufeng_njfy@163.com and E-Mail changliu@njnu.edu.cn 


\section{Introduction}

MicroRNAs (miRNAs) are a recently discovered class of short, non-coding, single stranded RNAs (approximately 22-24 nucleotides), and they function in transcriptional and post-transcriptional regulation of gene expression [1]. Mature miRNAs form part of an active RNA-induced silencing complex (RISC, also called microRNA ribonucleoprotein complex, miRNP) containing Dicer and many associated proteins [2]. This complex acts as a regulator of numerous biological processes by either triggering degradation of the target mRNAs or suppressing their translation through incomplete base-pairing to the 3' untranslated region (3'UTR) [3]. Although the functioning patterns and target genes of most miRNAs are still unknown, diverse miRNAs have recently been reported to participate in processes such as embryogenesis, embryonic development, stem cell pluripotency, differentiation, organogenesis, growth, cell proliferation and apoptosis [4-9].

Early embryonic development is a highly complicated process that comprises multitudinous biological events. Epigenetic regulation, considered to be one of the most important mechanisms, plays an important role in early embryonic development. Many studies have revealed that genomic DNA methylation is a dynamic process of reprogramming that mainly includes a wide range of genomic DNA demethylation statuses after fertilization and de novo methylation restored not long after implantation [10-12]. A previous study suggested that several miRNAs affect mouse embryonic development via DNA methylation during the morula to blastocyst transition [13].

In our previous study, miR29b was identified in mouse early embryos through miRNA microarray analysis (data not shown). Biological databases suggest that miR29b is highly conserved in evolution. To date, few studies have examined the role of miR29b in early embryonic development, although there are many reports that miR29b plays a role in DNA methylation by regulating Dnmt3a/3b expression [14-16]. In the present study, the expression patterns of miR29b and Dnmt3a/3b were analyzed in mouse early embryos at different stages, and the functional exploration of miR29b suggests an important role for miR29b in mouse early embryonic development mediated by miR29b-DNMT3 signaling.

\section{Materials and Methods}

Reagents and Animals

Reagents used for in vitro culture were from Sigma Chemicals (St. Louis, MO, USA) unless stated otherwise. All experimental animals (ICR white mice) were maintained in a controlled environment of $20-22^{\circ} \mathrm{C}$ with $50-70 \%$ humidity, 12/12-h light/dark cycle and adequate supply of food and water. Animal care and experimental procedures were conducted in accordance with the Animal Research Committee guidelines of Nanjing Medical University (China).

\section{Embryo Collection and Culture}

For zygote collection, 8-week-old female ICR mice were injected intraperitoneally (i.p.) with pregnant mare serum gonadotropin (PMSG; $10 \mathrm{IU}$ ) and human chorionic gonadotropin (HCG; $10 \mathrm{IU}$ ) $48 \mathrm{~h}$ later, followed by mating with male ICR mice. After 15-17 h, zygotes were obtained from the oviducts and placed into Hepes-buffered CZB medium. Cumulus cells were dispersed with $1 \mathrm{mg} / \mathrm{ml}$ hyaluronidase in Hepes-CZB. Cumulus-free zygotes were washed with Hepes-CZB medium and then cultured in CZB medium at $37^{\circ} \mathrm{C}$ in a humidified atmosphere of 5\% CO2 until the later stages of early embryos.

Immunofluorescence

Early embryos from zygote to blastocyst stage were fixed in $4 \%$ paraformaldehyde (PFA) in phosphatebuffered saline (PBS; pH 7.4) for approximate $30 \mathrm{~min}$ at room temperature (RT). After being treated in permeabilization buffer (0.5\% Triton X-100 in $20 \mathrm{mM}$ Hepes [pH 7.4], $3 \mathrm{mM} \mathrm{MgCl2,} 50 \mathrm{mM} \mathrm{NaCl}, 300 \mathrm{mM}$ sucrose, and $0.02 \% \mathrm{NaN} 3$ ) for $30 \mathrm{~min}$ at $37^{\circ} \mathrm{C}$, the embryos were blocked in $1 \%$ bovine serum albumin 
(BSA)-supplemented PBS for $1 \mathrm{~h}$ at RT and washed in PBS with 0.1\% Tween-20 three times for 5 min and then incubated at $4^{\circ}$ Covernight with 1:100 mouse monoclonal anti-Dnmt3a antibody (Cambridgeshire, UK, Abcam, ab13887), 1:100 mouse monoclonal anti-Dnmt3b antibody (Abcam, ab13604) or 1:100 Mouse monoclonal Anti-5-methylcytosine (5-mC) antibody (Abcam, ab10805). After being washed three times with PBS containing 0.1\% Tween-20 (5 min for each wash), the embryos were labeled with 1:100 FITCconjugated goat-anti-mouse IgG (Beijing ZhongShan Biotechnology) for $1 \mathrm{~h}$ at $37^{\circ} \mathrm{C}$. After being washed three times with PBS containing 0.1\% Tween-20, the embryos were co-stained with propidium iodide (PI; $10 \mu \mathrm{g} / \mathrm{ml}$ in PBS). Finally, embryos were mounted on glass slides with 1, 4-diazabicyclo [2.2.2] octane and examined under a ZEISS LSM 710 confocal laser scanning microscope (Carl ZEISS MicroImaging). ZEN (Zeiss Efficient Navigation) 2009 Light Edition software equipped with LSM Systems was used for data analysis as previously described [17-19]. The same parameters were used for microscopic analysis of the experimental and control groups.

\section{RNA (miRNA/mRNA) Isolation and Real-Time PCR}

Total RNA, including miRNAs and other small RNAs, was purified from early developmental stage embryos using a miRNeasy Mini Kit (Qiagen, 217004) according to the manufacturer's protocol. The cDNAs were reverse transcribed from total RNA samples using miR29b-specific, stem-loop RT primer from the TaqMan ${ }^{\circ}$ Small RNA Assays (Applied Biosystems, 4427975) and reagents from the TaqMan® MicroRNA Reverse Transcription Kit (Applied Biosystems, 4366596). Real-time PCR analysis was carried out using TaqMan ${ }^{\circledR}$ Universal Master Mix (Applied Biosystems, 4324018) using U6 snRNA as an endogenous control.

Otherwise, total RNA from early developmental stage embryos was extracted using the RNeasy Micro Kit (Qiagen, 74034). Real-time PCR analysis was carried out using SYBR ${ }^{\circledR}$ Premix EX Taq (Takara, DRR420A), and the gapdh gene acted as an internal control. The primer sequences used in the real-time PCR analysis were as follows: dnmt3a (sense): 5'-GAAGCGGAGTGAACCCCAAC-3' and (antisense): 5'-CCTTGGTCACACAGCAGCC-3'; dnmt3b (sense): 5'-GCCAGCCTCACGACAGGAAAC-3' and (antisense): 5'-GACTGGGGGTGAGGGAGCATC-3'; gapdh (sense): 5'-AGGTTGTCTCCTGCGACTTCA-3' and (antisense): 5'-GGGTGGTCCAGGGTTTCTTACT-3' The experiments were repeated at least three times.

\section{Microinjection of miR29b Inhibitor or Mimic into Zygotes}

The mirVana $®$ miRNA Inhibitor (Applied Biosystems, 4464084) or mirVana ${ }^{\circledR}$ miRNA Mimic (Applied Biosystems, 4464066) specific for mmu-miR29b (mouse miR29b) was microinjected into the cytoplasm of zygotes as described previously [20], and the mirVana ${ }^{\circledR}$ miRNA Inhibitor Negative Control \#1 (Applied Biosystems, 4464084) or mirVana $®$ miRNA Mimic Negative Control \#1(Applied Biosystems, 4464066) was used as the relevant negative control. These reagents (approximately 5-7 $\mu$ l per zygote) were microinjected at $100 \mathrm{nM}$, while non-injected zygotes served as the normal control groups. A Nikon Diaphot ECLIPSE TE 300 inverted microscope (Nikon, Yuko, Japan), equipped with Narishige MM0-202N hydraulic three-dimensional micromanipulators (Narishige Inc., Tokyo, Japan) was used for these experiments. After microinjection, the zygotes were washed and cultured in CZB medium for observation of further embryonic development or collected to determine Dnmt3a/3b expression.

\section{Statistical Analysis}

The differences between treatments were analyzed bypairedStudent's t-tests. Values of $\mathrm{P}<0.05$ were considered statistically significant. All data are expressed as the mean \pm standard deviation.

\section{Results}

Expression Patterns of miR29b and Dnmt3a/3b in Mouse Early Embryos

Real-time PCR analysis revealed the expression pattern of miR29b and Dnmt3a/3b in mouse early embryos at different stages (Fig. 1). Mouse miR29b was expressed at extremely high levels at the 2-cell stage, then decreased from the 4-cell stage, and maintained a 


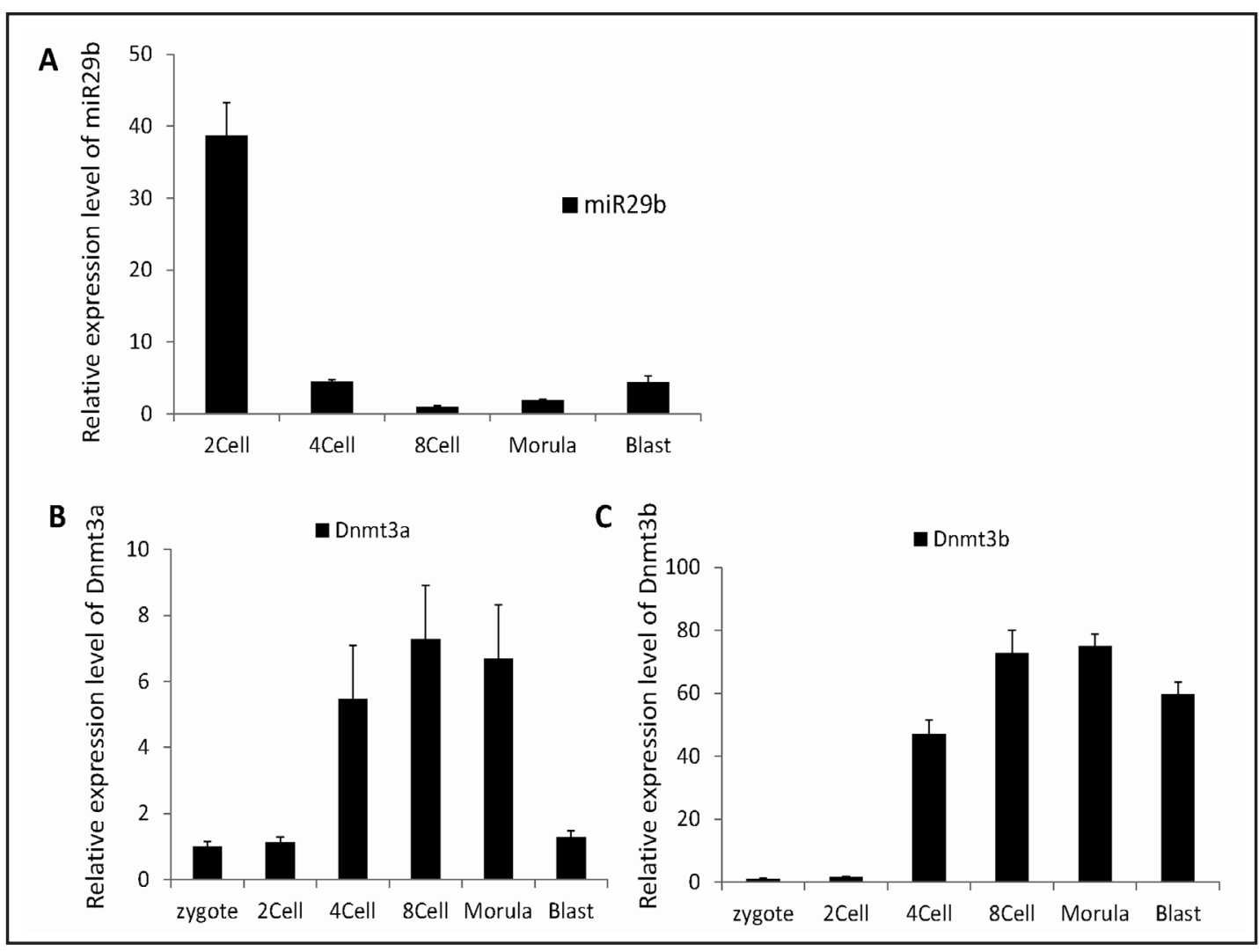

Fig. 1. Expression patterns of miR29b and Dnmt3a/3b in mouse early embryos by real-time PCR. (A) Expression of mouse miR29b was extremely high at the 2-cell stage, then decreased from the 4-cell stage, and was maintained at a relatively low level thereafter. U6 snRNA was used as the endogenous control. (B) Dnmt3a mRNA levels increased from the 4-cell stage, and then reverted to those of the 2-cell stage. (C) Dn$\mathrm{mt3b}$ mRNA levels also increased appreciably from the 4-cell stage and were subsequently maintained at a relatively high level to the blastocyst stage. Gapdh was used as the internal control. Data are expressed as the mean \pm SD for 3 replicates.

relatively low level thereafter (Fig. 1A). Meanwhile, the mRNA expression levels of both Dnmt3a and Dnmt3b mRNA increased significantly from the 4-cell stage (Fig. 1B, 1C), although Dnmt3a reverted to a low level at the blastocyst stage (Fig. 1B), while Dnmt3b was maintained at a relatively high level in subsequent stages until the blastocyst stage (Fig. 1C). These results confirmed the expression of miR29b and Dnmt3a/3b in mouse early embryos. It is noteworthy that Dnmt3a/3b expression levels exhibited an inverse correlation with miR29b, which suggested that these molecules are targets of miR29b and are negatively regulated by miR29b in mouse early embryos.

\section{Localization of DNMT3A/3B in Mouse Early Embryos}

The dynamic distribution of DNMT3A/3B proteins from the zygote to blastocyst stage is shown in Fig. 2. Immunofluorescence staining of DNMT3A/3B was observed predominantly in the nucleoplasm of embryos. As with the mRNA expression, there were differences between the expression of DNMT3A and DNMT3B proteins in the early embryo stages. DNMT3A protein expression decreased obviously in the morula stage, with almost no signal detected in the blastocyst stage (Fig. 2A), while DNMT3B was expressed persistently until the blastocyst stage, with expression increasing from the morula stage to the blastocyst stage (Fig. 2B). No significant differences were observed in the signals detected in the negative control (rabbit IgG) group at all stages of mouse early embryo development. 
Fig. 2. Subcellular localization of DNMT3A/3B in mouse early embryos. (A) Confocal microscopy image showing immunostaining of DNMT3A (green) and DNA (red) from the zygote to the blastocyst stage. (B) Immunostaining of DNMT3B (green) and DNA (red) from the zygote to the blastocyst stage.

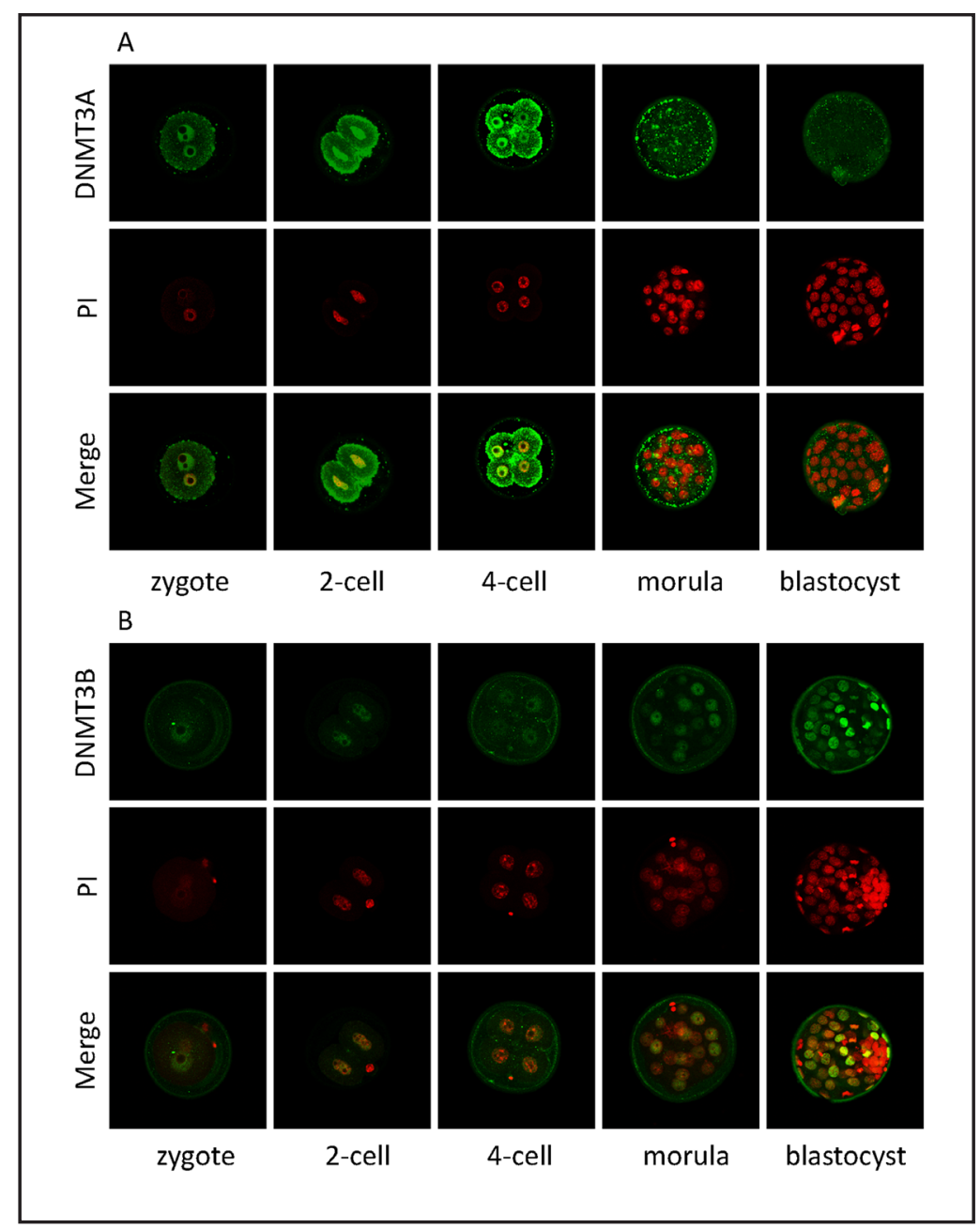

Alterations in miR29bActivity in Mouse Early Embryos

MiR29b regulates Dnmt3a/3bexpression in mouse early embryos. Dnmt3a/3b mRNA expression levels were detected using real-time PCR. Dnmt3a/3b mRNA expression increased markedly in miR29b inhibitor-injected embryos (collected from the 4-cell to blastocyst stage) compared with those in the negative control inhibitor-injected or non-injected embryos (Fig. $3 \mathrm{~A}, 3 \mathrm{C}$ ). In contrast to the effects of miR29b inhibition, both Dnmt3a and Dnmt3b mRNA levels decreased distinctly in miR29b-mimic-injected embryos (collected from the 4-cell to blastocyst stage) compared with those in the negative control mimic-injected or noninjected embryos (Fig. 3B, 3D). These results revealed that Dnmt3a/3b expression levels were regulated by miR29b in mouse early embryos.

Alterations in miR29b activity lead to early embryonic developmental blockade. To investigate the function of miR29b in mouse early embryonic development, a specific miR29b inhibitor or mimic was used to alter its activity in mouse early embryos. A remarkable block were observed from the morula stage (Day 3.5) to the blastocyst stage (Day 4.5) as a result of down-regulated miR $29 \mathrm{~b}$ activity, with only $50 \%$ to $60 \%$ of embryos progressing to the blastocyst stage compared to the negative and non-injected groups (Fig. 4A; Fig. 5A). Intriguingly, a similar phenotype observed in the miR29b mimic-injected groups compared with the negative and non-injected groups from the morula to the blastocyst stage (Fig. 4B; Fig. 5B), which suggested that up-regulation of miR29b also resulted in early embryonic developmental hysteresis in mouse. To access whether DNA methylation is affected by alterations in miR29b activity, we detected $5 \mathrm{mc}$ signal using immunofluorescence. 

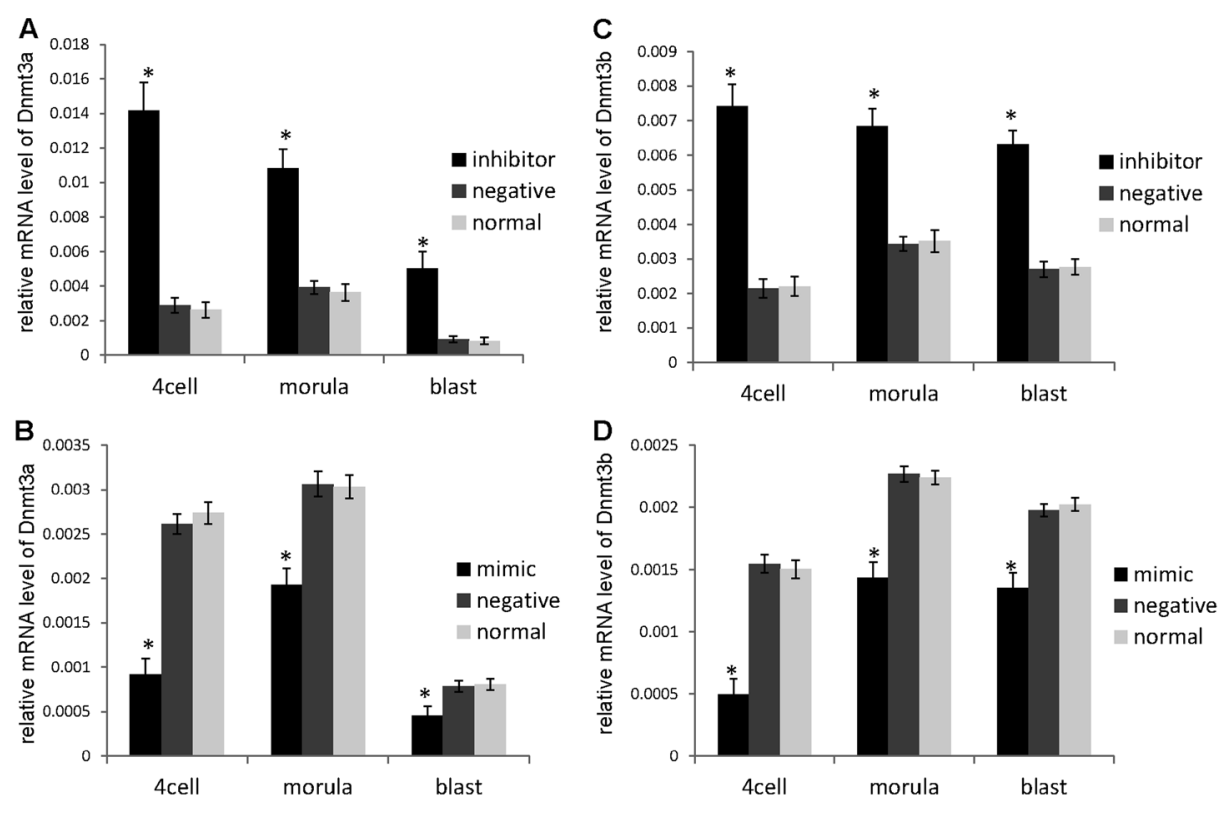

Fig. 3. Dnmt3a/3b expression was regulated by miR29b in mouse early embryos. (A, C) The expression levels of Dnmt3a/3b increased in miR29b inhibitor-injected embryos (collected from 4-cell to blast stage), compared with negative control inhibitor (labeled as negative) and non-injected groups (labeled as normal). (B, D) Dnmt3a/3b mRNA levels were significantly lower in miR29b-mimic microinjected embryos (collected from 4-cell to blast stage) compared with those in the negative control mimic (labeled as negative) and non-injected groups (labeled as normal). Data are expressed as the ratio of $2(-\Delta \Delta \mathrm{Ct})$ values relative to gapdh. Data represent the mean \pm SD of three real-time PCR experiments, ${ }^{*} \mathrm{P}<0.05$.

Fig. 4. Alteration of miR29b expression caused developmental retardation in mouse early embryos. (A) Down-regulation of miR29b by microinjection of an effective miR29b inhibitor resulted in retardation of early embryonic development. A significant growth-inhibitory effect was observed from the morula stage to the blastocyst stage. Only $50-60 \%$ of morula embryos progressed to the blastocyst stage compared with the rate observed in the negative and non-injected groups. (B) Up-regulation of miR29b by microinjection of an effective miR29b mimic also caused early embryonic developmental hysteresis. More than 50\% of embryos failed to develop to the blastocyst stage, in comparison with the negative and non-injected groups. Data represent the mean $\pm \mathrm{SD} ;{ }^{*} \mathrm{P}<0.01$.

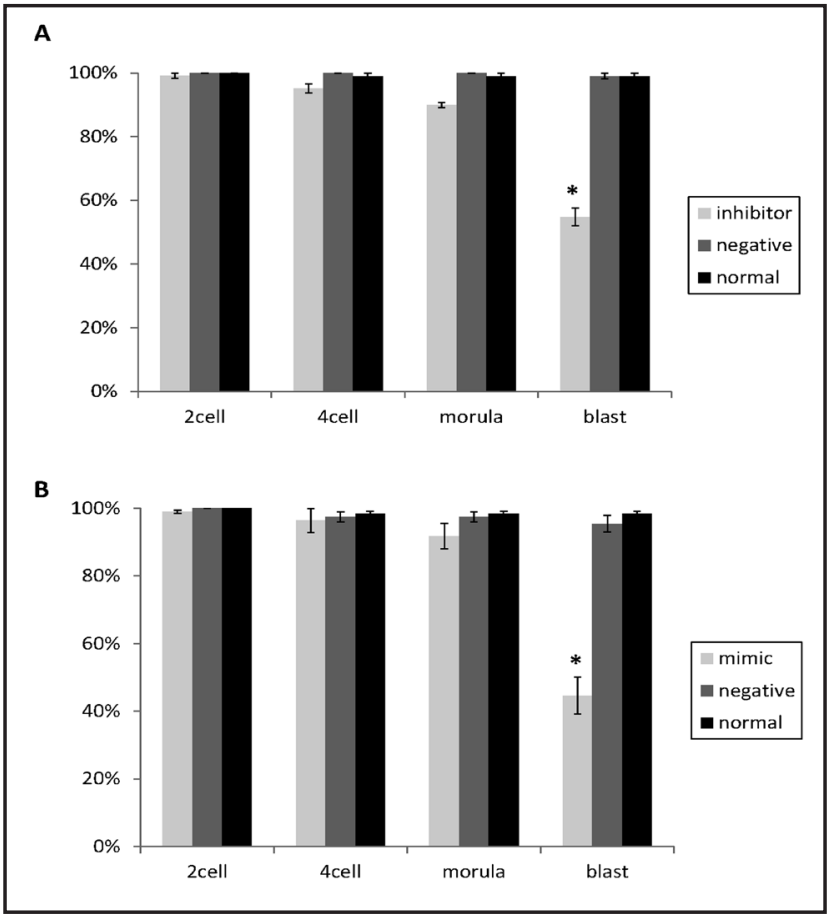

Interestingly, we find that mimic group significantly reduce DNA methylation level, but inhibitor group obviously increase DNA methylation level (Fig. 6). In summary, alterations 
Fig. 5. Embryonic development was blocked at the blastocyst stage following microinjection of the miR29b inhibitor or mimic. (A) Embryos injected with the miR29b inhibitor compared with those injected with the negative and normal (noninjected) groups at the blastocyst stage. (B) Embryos injected with the miR29b mimic compared with the negative and normal (non-injected) groups at the blastocyst stage.
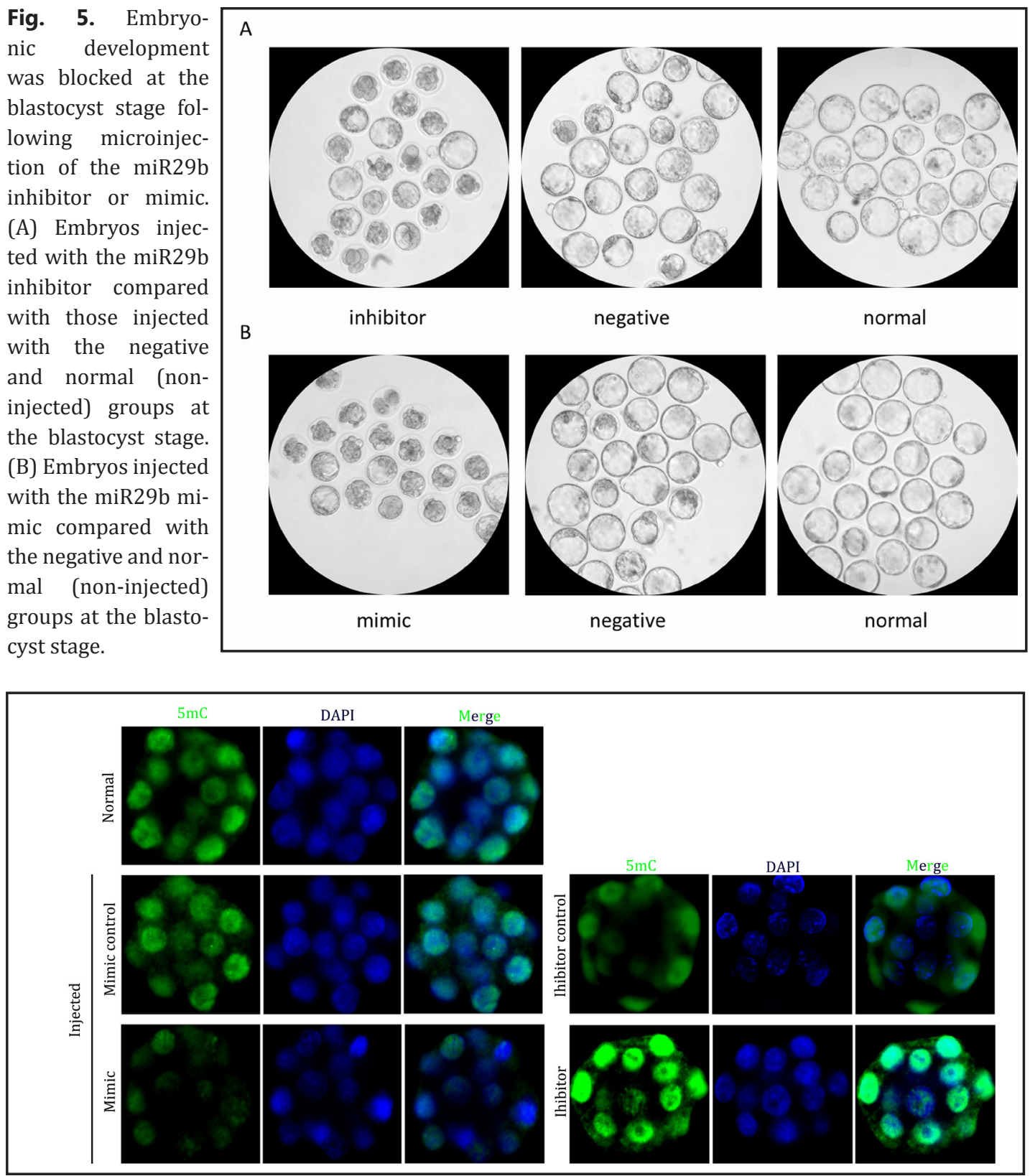

Fig. 6. Immunofluorescence of 5-methylcytosine $(5-\mathrm{mC})$ at the morula stage. Embryos were treated with a miR29b inhibitor or mimic and collected at the morula stage. Compared with the negative control and normal group, the 5-mC signal was significantly reduced in the mimic group but was noticeably increased in the inhibitor group.

in miR29b activity can cause to DNA methylation disorder and lead to early embryonic developmental blockade by regulating expression of DNMT3a/b. Functional analysis of miR29b revealed that miR29b activity influenced mouse early embryonic development.

\section{Discussion}

MiRNAs are small, non-coding RNAs that regulate the expression of many genes. Recent studies suggest that miRNAs could play crucial roles in the maternal-to-embryonic transition (MET), as expression profiles indicate a potential regulatory role during early developmental

\section{KARGER}


steps [21]. In previous studies, the miR29b gene was shown to be up-regulated in 2-cell embryos and was expressed at significantly reduced levels in developmentally retarded embryos compared with those in normal embryos. These results indicated that miR29b plays an important role in the process of embryonic development.

DNA methylation is widespread in most species and is crucial for gene expression and embryogenesis. Previous studies have shown that miR29 expression is inversely correlated to Dnmt3a/3b expression. The enforced expression of miR29s in lung cancer cell lines restores normal patterns of DNA methylation, induces re-expression of methylation-silenced tumor suppressor genes and inhibits tumorigenicity both in vitro and in vivo [22]. Current studies show that overexpression of miR29b in acute myeloid leukemia cells results in marked reduction in the expression of the DNA methyltransferases, DNMT1, DNMT3A and DNMT3B, at both the RNA and protein levels [23].

In the present study, we first characterized the role of miR29b in the regulation of DNA methylation during early embryo development in the mouse. Our data showed upregulation of miR29b in the 2-cell embryos, with low expression levels maintained during the period from the 4-cell embryo stage to the blastocyst stage. In contrast, the expression of Dnmt3a and Dnmt3b were upregulated from the 4-cell embryo stage, with high expression levels maintained through later time points. Immunofluorescence studies showed that the expression of DNMT3A and DNMT3B proteins reflected a similar pattern to that observed for Dnmt3a and Dnmt3b mRNA expression. These results are consistent with previous reports. Until this work, the function and mechanisms of miR29b in the process of preimplantation embryonic development had not been reported. Additionally, we used knock-down and over-expression experiments to further study the functional role and potential molecular mechanisms of mir29b in the process of embryonic development. According to a previous report, the loss of DNMTs leads to a phenotype that includes developmental arrest or death of the pups shortly after birth, due to DNA hypomethylation [24]. Our study showed that up-regulation of miR29b resulted in a marked reduction of the expression of Dnmt3a and Dnmt3b at the mRNA level and DNA hypomethylation in the embryo. Conversely, DNA methylation could contribute to gene silencing [25]. DNA hypermethylation might inhibit some of the genes which are involved in embryo development. Although the loci of DNA methylation, and the genes involved in this process, are still unknown, our data suggest that homeostasis of DNA methylation is related to embryo development. Taken together, miR29b may contribute to disruption of DNA methylation by regulating expression of DNMT3a/b, which leads to early embryonic developmental blockade. These results further validate that miR29b plays an important role in the development of early embryos through regulating the expression of the Dnmt3a/Dnmt3b gene. In addition, our results suggest that precise methylation regulation is essential for mouse early embryonic development.

Using various experimental methods, certain miRNAs were continuously reported in recent studies in the field of animal embryogenesis [26-30]. During the implantation period, potential regulators of endometrial receptivity include miRNAs [31]. These findings all indicate that miRNAs play a critical role in embryo development. During mammalian development, the methylation patterns required for genomic imprinting change dynamically. DNA methylation, including maintenance methylation and de novo methylation directed by DNA methyltransferases (DNMTs), is important for embryo development. Abnormal function of these DNMTs may have serious consequences for embryonic development, such as inhibition of DNA methylation maintenance, leading to a decreased implantation rate of embryos, increased fetal absorption, and poor fetal and placental development [32]. In summary, our data suggest that miR29b takes part in the development of mouse preimplantation embryos by regulating the expression of DNMT3A and DNMT3B. These results represent a theoretical basis for clarification of the mechanisms underlying the regulation of embryonic development. 
Zhang et al.: MiR-29b in Mouse Early Embryos

\section{Acknowledgments}

This study was financially supported by the National Natural Science Foundation of China (81100420, 81270701, and 81471457).

\section{Disclosure Statement}

The authors declare that they have no competing interests.

\section{References}

1 Chen K, Rajewsky N: The evolution of gene regulation by transcription factors and micrornas. Nat Rev Genet 2007;8:93-103.

2 Rana TM: Illuminating the silence: Understanding the structure and function of small rnas. Nat Rev Mol Cell Biol 2007;8:23-36.

3 Wu L, Fan J, Belasco JG: Micrornas direct rapid deadenylation of mrna. Proc Natl Acad Sci U S A 2006;103:4034-4039.

4 Amanai M, Brahmajosyula M, Perry AC: A restricted role for sperm-borne micrornas in mammalian fertilization. Biol Reprod 2006;75:877-884.

5 Tang F, Hajkova P, Barton SC, Lao K, Surani MA: Microrna expression profiling of single whole embryonic stem cells. Nucleic Acids Res 2006;34:e9.

6 Cui XS, Shen XH, Kim NH: Dicer1 expression in preimplantation mouse embryos: Involvement of oct3/4 transcription at the blastocyst stage. Biochem Biophys Res Commun 2007;352:231-236.

7 Byrne MJ, Warner CM: Microrna expression in preimplantation mouse embryos from ped gene positive compared to ped gene negative mice. J Assist Reprod Genet 2008;25:205-214.

8 Tzur G, Levy A, Meiri E, Barad O, Spector Y, Bentwich Z, Mizrahi L, Katzenellenbogen M, Ben-Shushan E, Reubinoff BE, Galun E: Microrna expression patterns and function in endodermal differentiation of human embryonic stem cells. PloS one 2008;3:e3726.

-9 Renthal NE CC, Williams KC, Gerard RD, Prange-Kiel J,Mendelson CR: Mir-200 family and targets, zeb1 and zeb2, modulate uterine quiescence and contractility during pregnancy and labor. Proc Natl Acad Sci U S A 2010;107:20828 - 20833.

10 Watanabe D SI, Tada T, et al.: Stage- and cell-specific expression of dnmt3a and dnmt3b during embryogenesis. Mech Dev 2002;118:187-190.

11 Hu YG, Hirasawa R, Hu JL, Hata K, Li CL, Jin Y, Chen T, Li E, Rigolet M, Viegas-Pequignot E, Sasaki H, Xu GL: Regulation of DNA methylation activity through dnmt3l promoter methylation by dnmt3 enzymes in embryonic development. Hum Mol Genet 2008;17:2654-2664.

12 Santos F, Hendrich B, Reik W, Dean W: Dynamic reprogramming of DNA methylation in the early mouse embryo. Dev Biol 2002;241:172-182.

13 Lee YM, Chen HW, Maurya PK, Su CM, Tzeng CR: Microrna regulation via DNA methylation during the morula to blastocyst transition in mice. Mol Hum Reprod 2012;18:184-193.

14 Takada S, Berezikov E, Choi YL, Yamashita Y, Mano H: Potential role of mir-29b in modulation of dnmt3a and dnmt3b expression in primordial germ cells of female mouse embryos. Rna 2009;15:1507-1514.

15 Pandey M, Sultana S, Gupta KP: Involvement of epigenetics and microrna-29b in the urethane induced inception and establishment of mouse lung tumors. Exp Mol Pathol 2013;96:61-70.

16 Guo X, Liu Q, Wang G, Zhu S, Gao L, Hong W, Chen Y, Wu M, Liu H, Jiang C, Kang J: Microrna-29b is a novel mediator of sox2 function in the regulation of somatic cell reprogramming. Cell Res 2013;23:142-156.

$\checkmark 17$ Liu M, Hales BF, Robaire B: Effects of four chemotherapeutic agents, bleomycin, etoposide, cisplatin, and cyclophosphamide, on DNA damage and telomeres in a mouse spermatogonial cell line. Biol Reprod 2014;90:72.

18 Liu M, Shi X, Bi Y, Qi L, Guo X, Wang L, Zhou Z, Sha J: Shcbp1l, a conserved protein in mammals, is predominantly expressed in male germ cells and maintains spindle stability during meiosis in testis. Mol Hum Reprod 2014;20:463-475. 
19 Liu M, Qi L, Zeng Y, Yang Y, Bi Y, Shi X, Zhu H, Zhou Z, Sha J: Transient scrotal hyperthermia induces lipid droplet accumulation and reveals a different adfp expression pattern between the testes and liver in mice. PloS one 2012; 7:e45694.

20 Dai Y LC, Hutchings A, Sun Y, Moor R.: Selective requirement for cdc25c protein synthesis during meiotic progression. Biol Reprod 2000;62:519-532.

21 Mondou E, Dufort I, Gohin M, Fournier E, Sirard MA: Analysis of micrornas and their precursors in bovine early embryonic development. Mol Hum Reprod 2012;18:425-434.

-22 Fabbri M, Garzon R, Cimmino A, Liu Z, Zanesi N, Callegari E, Liu S, Alder H, Costinean S, FernandezCymering C, Volinia S, Guler G, Morrison CD, Chan KK, Marcucci G, Calin GA, Huebner K, Croce CM: Microrna-29 family reverts aberrant methylation in lung cancer by targeting DNA methyltransferases 3a and 3b. Proc Natl Acad Sci U S A 2007;104:15805-15810.

23 Garzon R, Liu S, Fabbri M, Liu Z, Heaphy CE, Callegari E, Schwind S, Pang J, Yu J, Muthusamy N, Havelange V, Volinia S, Blum W, Rush LJ, Perrotti D, Andreeff M, Bloomfield CD, Byrd JC, Chan K, Wu LC, Croce CM, Marcucci G: Microrna-29b induces global DNA hypomethylation and tumor suppressor gene reexpression in acute myeloid leukemia by targeting directly dnmt3a and $3 \mathrm{~b}$ and indirectly dnmt1. Blood 2009;113:6411-6418.

24 Li E: Chromatin modification and epigenetic reprogramming in mammalian development. Nat Rev Genet 2002;3:662-673.

-25 Paszkowski J, Whitham SA: Gene silencing and DNA methylation processes. Curr Opin Plant Biol 2001;4:123-129.

26 Li M XY, Gu Y, Zhang K: Micrornaome of porcine pre- and postnatal development. PloS one 2010;5

-27 Castro FO, Sharbati S, Rodriguez-Alvarez LL, Cox JF, Hultschig C, Einspanier R: Microrna expression profiling of elongated cloned and in vitro-fertilized bovine embryos. Theriogenology 2010;73:71-85.

28 Mineno J, Okamoto S, Ando T, Sato M, Chono H, Izu H, Takayama M, Asada K, Mirochnitchenko O, Inouye M, Kato I: The expression profile of micrornas in mouse embryos. Nucleic Acids Res 2006;34:1765-1771.

29 Darnell DK, Kaur S, Stanislaw S, Konieczka JH, Yatskievych TA, Antin PB: Microrna expression during chick embryo development. Dev Dyn 2006;235:3156-3165.

30 Wienholds E, Kloosterman WP, Miska E, Alvarez-Saavedra E, Berezikov E, de Bruijn E, Horvitz HR, Kauppinen S, Plasterk RH: Microrna expression in zebrafish embryonic development. Science 2005;309:310-311.

-31 Revel A, Achache H, Stevens J, Smith Y, Reich R: Micrornas are associated with human embryo implantation defects. Hum Reprod 2011;26:2830-2840.

-32 Yin LJ, Zhang Y, Lv PP, He WH, Wu YT, Liu AX, Ding GL, Dong MY, Qu F, Xu CM, Zhu XM, Huang HF: Insufficient maintenance DNA methylation is associated with abnormal embryonic development. BMC Med 2012;10:26. 